\title{
Variance-Based Interaction Index Measuring Heteroscedasticity
}

\author{
Keiichi Ito $^{\mathrm{a}, \mathrm{b}, *}$, Ivo Couckuyt ${ }^{\mathrm{a}}$, Silvia Poles $^{\mathrm{b}}$, Tom Dhaene $^{\mathrm{a}}$ \\ ${ }^{a}$ Department of Information Technology (INTEC), Ghent University - iMinds, Gaston Crommenlaan 8 bus 201, 9050 Ledeberg - Ghent, Belgium \\ ${ }^{b}$ Noesis Solutions, Gaston Geenslaan 11 B4, 3001 Leuven, Belgium \\ http://dx.doi.org/10.1016/j.cpc.2016.02.032
}

(c)2016. This manuscript version is made available under the CC-BY-NC-ND 4.0 license http://creativecommons.org/licenses/by-nc-nd/4.0/

\begin{abstract}
This work is motivated by the need to deal with models with high-dimensional input spaces of real variables. One way to tackle high-dimensional problems is to identify interaction or non-interaction among input parameters. We propose a new variance-based sensitivity interaction index that can detect and quantify interactions among the input variables of mathematical functions and computer simulations. The computation is very similar to first-order sensitivity indices by Sobol'. The proposed interaction index can quantify the relative importance of input variables in interaction. Furthermore, detection of non-interaction for screening can be done with as low as $4 n+2$ function evaluations, where $n$ is the number of input variables. Using the interaction indices based on heteroscedasticity, the original function may be decomposed into a set of lower dimensional functions which may then be analyzed separately.
\end{abstract}

Keywords: Sensitivity Analysis, Sobol’ Indices, Problem Decomposition, Interaction, Optimization, Parameter Estimation

\section{Introduction}

In today's engineering, computer simulations are widely used to understand the behavior of complex systems and to optimize their input variables to obtain satisfactory designs before actual physical prototypes are built. The simulators are usually black-box or too complex to render a mathematical approach feasible. Sensitivity analysis enables us to understand how the changes in input variables affect the variance of the output.

As a part of the sensitivity analysis, identifying interacting and additive-effect variables is important in design optimization and engineering analysis of black box models. Two input variables are said to interact if their effect on the output cannot be expressed as a sum of their single effects. If the variable is additive (noninteracting), that variable can be treated independently from other variables. Then, we can separate our effort between the analysis of the interacting part which is often the more subtle and difficult part, and the analysis of the additive effect part. In this study, we will treat a methodology to detect and quantify this interaction of input variables to deterministic black-box models.

\footnotetext{
${ }^{*}$ Corresponding author

Email address: itokeiic@gmail.com (Keiichi Ito)
}

A widely recognized way of quantifying interaction is by calculating the difference between total effect indices and first order sensitivity indices in variance-based global sensitivity analysis $[1,2,3]$. In practice, the effectiveness of this method hinges on the accuracy of the sensitivity indices, which may demand a very high number of Monte Carlo sampling.

On the other hand, there are one-at-a-time methods often used for screening important variables by estimating average partial derivative magnitudes of the outputs obtained from factor levels (i.e., sampling on grid points) or as perturbations of Monte Carlo samples $[4,5,6,7]$. In these methods, interaction effects are not distinguished from non-linear effects of a particular input variable $[4,5,7]$ or it is computed in a factorial design manner [6] with a preferred number of factors, for example $3,6,10,15, \ldots$

We propose an approach to decompose a highdimensional problem into a set of lower dimensional problems via novel interaction indices which use the heteroscedasticity of marginal distributions. Heteroscedasticity refers to the circumstance in which the variability of a variable is unequal across the range of values of a second variable (a factor) that predicts it. Calculation of these interaction indices is a simple 
extension to Sobol indices [8] and gives information about particular variable(s) being non-interacting or interacting with other variables. The method uses Monte Carlo integration, but is very robust against loss of accuracy even when the number of random samples is modest. Due to this property, the proposed method can be used for both quantification and screening of interaction among input variables depending on the computational budget.

In the following discussions, $E()$ denotes the expectation or the average value of the variable inside the bracket. Likewise, $V()$ denotes the variance. Sometimes, we put a subscript below $V$ to clarify the source of the variance. We also employ an indexing convention $-i$ to denote "all other indices except $i$ ". For example,

$$
\underset{x_{-i}}{V}\left(y \mid x_{i}\right)
$$

means variance of $y$ given $x_{i}$ (so the variance of y comes from the variance of sources other than $x_{i}$, thus $\underset{x_{-i}}{V}$ ).

\section{Sobol' Indices and High-Dimensional Model Rep- resentation (HDMR)}

Consider a deterministic model

$$
y=f(\mathbf{x})
$$

where $\mathbf{x}=\left(x_{1}, x_{2}, \ldots, x_{n}\right)$ is a vector of $n$ input variables and $y$ is the model output. $f(\mathbf{x})$ can be decomposed into a form referred to as the high-dimensional model representation.

$$
\begin{aligned}
f(\mathbf{x})= & f_{0}+\sum_{i} f_{i}\left(x_{i}\right)+\sum_{i<j} f_{i j}\left(x_{i}, x_{j}\right) \\
& +\sum_{i<j<k} f_{i j k}\left(x_{i}, x_{j}, x_{k}\right)+\ldots
\end{aligned}
$$

This decomposition of the function is not unique as the lower order can be selected arbitrarily and the highest order term can be written as the difference between $f(\mathbf{x})$ and the lower order terms. However, if the average of each of the term in the summands of the right hand side of equation (1) is set to zero (e.g., $\int f_{i}\left(x_{i}\right) d x_{i}=0$ ) and $f_{0}$ is set to be a constant, the expression is proven to be unique [8]. The terms are given as follows:

$$
\begin{aligned}
f_{0}= & E(y) \\
f_{i}\left(x_{i}\right)= & E\left(y \mid x_{i}\right)-f_{0} \\
f_{i j}\left(x_{i}, x_{j}\right)= & E\left(y \mid x_{i}, x_{j}\right)-f_{i}\left(x_{i}\right) \\
& -f_{j}\left(x_{j}\right)-f_{0} \\
f_{i j k}\left(x_{i}, x_{j}, x_{k}\right)= & E\left(y \mid x_{i}, x_{j}, x_{k}\right)-f_{i j}\left(x_{i}, x_{j}\right) \\
& -f_{i k}\left(x_{i}, x_{k}\right)-f_{j k}\left(x_{j}, x_{k}\right) \\
& -f_{i}\left(x_{i}\right)-f_{j}\left(x_{j}\right)-f_{k}\left(x_{k}\right) \\
& -f_{0} .
\end{aligned}
$$

The first order sensitivity index for variable $x_{i}$ is given by

$$
S_{i}=\frac{\underset{x_{i}}{V}\left[E\left(y \mid x_{i}\right)\right]}{V(y)},
$$

and if we calculated the indices to the highest order, we have

$$
\sum_{i=1}^{n} S_{i}+\sum_{i=1}^{n} \sum_{j=i+1}^{n} S_{i j}+\ldots+S_{1 \ldots n}=1 .
$$

The $S_{i \in\{1,2, \ldots, n\}}$ are called first-order Sobol' indices or sensitivity indices $[2,9]$.

The total effect index [1] includes interaction effects in addition to the first-order sensitivity indices, and can be defined as

$$
S_{T i}=1-S_{-i},
$$

where $S_{-i}$ signifies the sum of all the sensitivity indices except those that include variances due to $x_{i}$. For example, if $i \in\{1,2,3\}$, the total effect index of $x_{1}$ is

$$
\begin{aligned}
S_{T 1} & =S_{1}+S_{12}+S_{13}+S_{123} \\
& =1-S_{2}-S_{3}-S_{23} .
\end{aligned}
$$

The total effect index defined in equation (8) is useful in variable screening. Variables with $S_{T i} \simeq 0$ can be held constant at an arbitrary value within its lower and upper bounds since it means that the variable's value does not contribute to the variance in the output ${ }^{1}$. The first order sensitivity indices in equation (6) alone cannot be used for this purpose if there is a significant amount of interactions among the variables.

\section{Computation}

We now formulate a way to compute the first order sensitivity indices. It is also assumed that the function $f(\mathbf{x})$ is square integrable in $\mathbf{x} \in \Omega$ where $\Omega$ is a

\footnotetext{
${ }^{1}$ Strictly speaking, this holds only to a probability [8, Theorem 2].
} 
$n$-dimensional domain of integration of real variables. Uniform distributions are assumed on the inputs, and inputs are uncorrelated with each other. The total variance is therefore

$$
\begin{aligned}
D & =\underset{\mathbf{x}}{V}(f(\mathbf{x})) \\
& =\frac{1}{\mathcal{V}} \int_{\mathbf{x} \in \Omega} f^{2}(\mathbf{x}) d \mathbf{x}-f_{0}^{2},
\end{aligned}
$$

where $\mathcal{V}=\int_{\mathbf{x} \in \Omega} d \mathbf{x}$ and $d \mathbf{x}=d x_{1} d x_{2} \ldots d x_{n}$. The average of $f(\mathbf{x})$ is given by

$$
f_{0}=\frac{1}{\mathcal{V}} \int_{\mathbf{x} \in \Omega} f(\mathbf{x}) d \mathbf{x} .
$$

The multidimensional integral of equation (10) can be computed using Monte Carlo integration. Similarly,

$$
f_{i}\left(x_{i}\right)=\frac{1}{\mathcal{V}_{-i}} \int_{\mathbf{x} \in \Omega_{-i}} f\left(x_{1}, x_{2}, \ldots, x_{n}\right) d x_{-i}-f_{0},
$$

where $d x_{-i}=d x_{1} d x_{2} \ldots d x_{i-1} d x_{i+1} \ldots d x_{n}, \Omega_{-i}$ is the domain of integration with $x_{i}$ fixed, and

$$
\mathcal{V}_{-i}=\int_{\mathbf{x} \in \Omega_{-i}} d x_{-i}
$$

We also define $V\left(y \mid x_{i}\right)$ for later use in our discussion,

$$
\begin{aligned}
& \underset{x_{-i}}{V\left(y \mid x_{i}\right)=} \\
& \quad \frac{1}{\mathcal{V}_{-i}} \int_{\mathbf{x} \in \Omega_{-i}} f^{2}\left(x_{1}, x_{2}, \ldots, x_{n}\right) d x_{-i}-f_{0}^{2} .
\end{aligned}
$$

This is the variance of $y$ when $x_{i}$ is fixed at a certain value. Again, in equations (11) and (12), the integrations are performed using the Monte Carlo method, but this time $x_{i}$ is held constant. By fixing $x_{i}$ at various values, we can conduct the next integration to obtain $V\left[E\left(y \mid x_{i}\right)\right]$.

$$
D_{i}=\underset{x_{i}}{V}\left[E\left(y \mid x_{i}\right)\right]=\underset{x_{i}}{V}\left(f_{i}\left(x_{i}\right)\right)=\frac{1}{\mathcal{V}_{i}} \int_{x_{i} \in \Omega_{i}} f_{i}^{2}\left(x_{i}\right) d x_{i}
$$

where $\Omega_{i}$ is the domain of integration for $x_{i}$, and $\mathcal{V}_{i}$ is the domain interval length of $x_{i}$. Then,

$$
S_{i}=\frac{D_{i}}{D}
$$

The computation of $f_{i}\left(x_{i}\right)$ at different values of $x_{i}$ to calculate $D_{i}$ in equation (13) is a brute-force approach. It requires $m \times(n \times l+1)$ function evaluations, where $m$ is the number of Monte Carlo samples, $n$ is the number of input variables, and $l$ is the number of different $x_{i}$ values that are used to compute equation (13). There is a more efficient method in which all $S_{i}$ and $S_{T i}$ are calculated in $m \times(n+2)$ function evaluations [10] provided that all input variables' distributions are independent.

Note that estimators have been recently developed to extend [10] to the case of correlated and dependent inputs $[11,12,13]$

\section{Interactions in Reliability and Optimization}

In the process of optimization, for example, minimizing $y$ by judicious choice of $x_{i}$, one would also be interested in the variance of $y$ given $x_{i}, V\left(y \mid x_{i}\right)$ or more generally, the distribution of $y$ given $x_{i}$. Let us denote such distribution (or probability density function) as $p\left(y \mid x_{i}\right)$. This information can easily be obtained during the calculation of the first order Sobol' Indices. This information can be used in three ways. First, it tells you for what value of $x_{i}$ one could possibly have the smallest $y$. Second, it tells you if $x_{i}$ has any interaction with other variables. Finally, it tells you what value of $x_{i}$ would satisfy certain reliability criteria. That is, one could draw a threshold value for $y$ beyond which these variances should not exceed.

If some or all of the $x_{i}$ contain uncertainties such that their intervals cannot be reduced beyond a certain level, the resulting $p\left(y \mid x_{i}\right)$ s will represent the uncertainties in the output due to the uncertainties in these $x_{i}$. For reliability purposes, one may also be interested in $\max \left(y \mid x_{i}\right)$ which is the maximum $y$ (that occurred in Monte Carlo simulations) given $x_{i}$.

Figure 1 shows an example of representing $p\left(y \mid x_{i}\right)$ as box plots. The example shows the spreads of two outputs $y_{o}, o \in\{1,2\}$ i.e. $p\left(y_{o} \mid x_{i}\right)$ in vertical axes with respect to three input variables $x_{1}, x_{2}$, and $x_{3}$. We see by visual inspection that $y_{1}$ is composed of purely additive effects from $x_{1}, x_{2}$ and $x_{3}$ because all the spreads of $p\left(y_{1} \mid x_{1}\right), p\left(y \mid x_{2}\right)$ and $p\left(y \mid x_{3}\right)$ as shown by the box sizes are constant across different values of $x_{1}, x_{2}$ and $x_{3}$, respectively (i.e. Homoscedastic behavior). If $x_{i}$ produces an additive effect in the output, it should only cause a shift in the mean of $p\left(y \mid x_{i}\right)$ according to equation (1).

On the other hand, $x_{2}$ and $x_{3}$ have interactions in $y_{2}$ because the spreads of $p\left(y_{2} \mid x_{2}\right)$ and $p\left(y_{2} \mid x_{3}\right)$ are not constant. If the output is determined only by the three inputs, we can conclude that $x_{2}$ and $x_{3}$ interact with each other in $y_{2}$. The quadratic effect $x_{1}$ to $y_{2}$ is additive since the $p\left(y_{2} \mid x_{1}\right)$ stays constant. If a variable does not interact, it can be treated independently, with other variables fixed or vice versa, without any loss of information. 
In the above example, the marginal spread of an output $p\left(y \mid x_{i}\right)$ was expressed as box plots as one would get from the brute-force approach, but the marginal scatter plot of $y$ vs. $x_{i}$ as one would obtain from the efficient computations [10] can also be informative for the three purposes above.

\section{Interaction Indices}

In order to quantify the interaction of input variables, we propose the following interaction index,

$$
I_{i}^{2}=\frac{\underset{x_{i}}{V}\left[\underset{x_{-i}}{\left.V\left(y \mid x_{i}\right)\right]}\right.}{V^{2}(y)},
$$

or its square root form,

$$
I_{i}=\frac{\sqrt{\begin{array}{l}
V \\
x_{i}
\end{array}\left[\begin{array}{c}
\left.V\left(y \mid x_{i}\right)\right] \\
x_{-i}
\end{array}\right.}}{V(y)},
$$

where we can compute $V_{x_{-}}\left(y \mid x_{i}\right)$ from equation (12). We can then set a threshold $\varepsilon$ below which we say that the input $x_{i}$ does not have significant interaction with other input variables and thus can be treated independently. Note that the interaction index $I_{i}$ is domain dependent. Even if the underlying function is the same, different $\Omega$ produce different values of $I_{i}$ in general. For example, two input variables $x_{i}$ and $x_{j}$, with $i \neq j$, may be interacting if varied substantially but may be non-interacting if varied by a small amount around certain points. The $\varepsilon$ is typically very small near the arithmetic precision. Mathematically speaking, $I_{i}=0$ for non-interacting input $x_{i}$ and $I_{i}>0$ for interacting $x_{i}$.

We can extend this concept to detect two and higher dimensional subproblems.

$$
\begin{aligned}
I_{i j}^{2} & =\frac{V\left[V\left(y \mid x_{i}, x_{j}\right)\right]}{V^{2}(y)}, \\
I_{i j k}^{2} & =\frac{V\left[V\left(y \mid x_{i}, x_{j}, x_{k}\right)\right]}{V^{2}(y)}, \\
& \ldots
\end{aligned}
$$

The indices $I_{i j}$ can be interpreted as follows. Let $i$ and $j$ be the indices whose input variable has shown to have interaction with other input variables: $I_{i}>\varepsilon, I_{j}>\varepsilon$, and $i<j$. Then, $0 \leq I_{i j} \leq \varepsilon$ means that input combinations specified by $x_{i}$ and $x_{j}$ produce an additive effect to the output $y$. This means that there is no higher order interaction for this particular pair of input variables $x_{i}$ and $x_{j}$. In the HDMR expression in equation (1), it means that a term $f_{i j}\left(x_{i}, x_{j}\right)$ is not zero. On the other hand, $I_{i j}>\varepsilon$ implies second or higher order interactions exist with some other input variables. For $I_{i j k}$ and higher follows the same argument.

\section{The Basic Idea Step by Step}

To clarify the idea of using heteroscedasticity in detecting (non-)interactions, let us consider the following two equations

$$
\begin{aligned}
& y_{1}=x_{1}+x_{2}, \\
& y_{2}=x_{1} \cdot x_{2} .
\end{aligned}
$$

We will carry out the brute-force calculation of

$$
\underset{x_{i}}{V}\left[\underset{x_{-i}}{V}\left(y \mid x_{i}\right)\right]
$$

step by step. The calculation will be done with $m=2$ and $l=2$.

Let us start with the (contrived) two sample points given in Table 1. In Table 2, the sample points were

Table 1: Initial two samples

\begin{tabular}{rrrr}
\hline$x_{1}$ & $x_{2}$ & $y_{1}$ & $y_{2}$ \\
\hline 1 & 2 & 3 & 2 \\
3 & 4 & 7 & 12 \\
\hline
\end{tabular}

replaced with $x_{1}=1$ and in Table 3 with $x_{1}=3$.

Table 2: $x_{1}$ fixed at 1

\begin{tabular}{rrrr}
\hline$x_{1}$ & $x_{2}$ & $y_{1}$ & $y_{2}$ \\
\hline 1 & 2 & 3 & 2 \\
1 & 4 & 5 & 4 \\
\hline
\end{tabular}

Table 3: $x_{1}$ fixed at 3

\begin{tabular}{rrrr}
\hline$x_{1}$ & $x_{2}$ & $y_{1}$ & $y_{2}$ \\
\hline 3 & 2 & 5 & 6 \\
3 & 4 & 7 & 12 \\
\hline
\end{tabular}

From Table 2 and Table 3 we can calculate $\underset{x_{-1}}{V}\left(y_{1} \mid x_{1}\right)$ and $\underset{x_{-1}}{V}\left(y_{2} \mid x_{1}\right)$ at the two $x_{1}$ locations, namely 1 and 3 . These are tabulated in Table 4. Note that for $y_{1}$, its values were simply shifted by 2 if you compare Table 2 and Table 3. Thus, in Table 4, $V\left(y_{1} \mid x_{1}\right)$ are identical at both $x_{1}=1$ and at $x_{1}=3$. This is because the $x_{-1}$ ( $x_{2}$ in this case) values were identical in both tables and $x_{1}$ is an additive contribution for $y_{1}$. For $y_{2}$, the multiplicative 


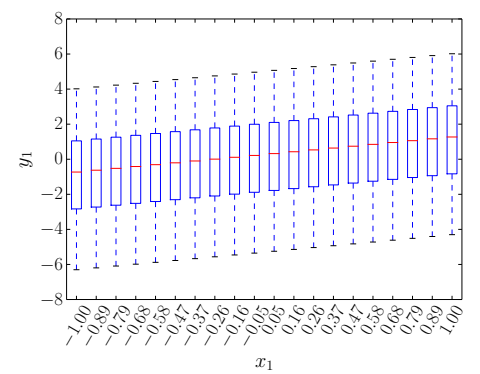

(a) Additive (Homoscedastic)

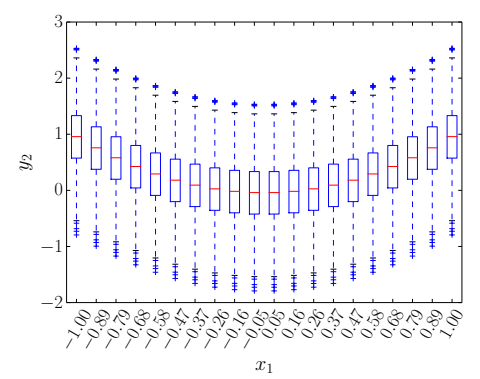

(d) Additive (Homoscedastic)

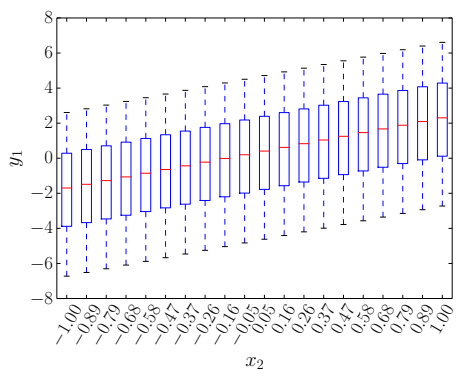

(b) Additive (Homoscedastic)

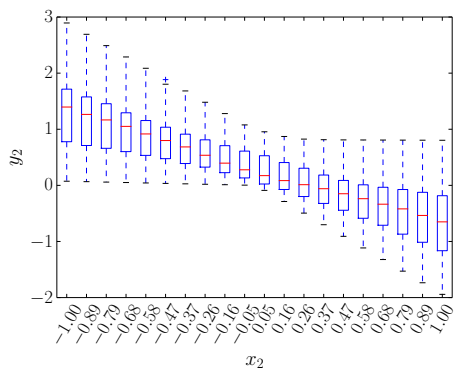

(e) Interacting (Heteroscedastic)

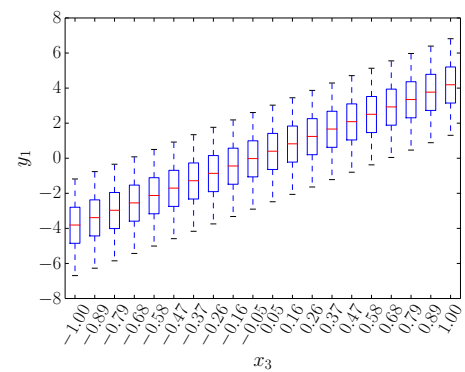

(c) Additive (Homoscedastic)

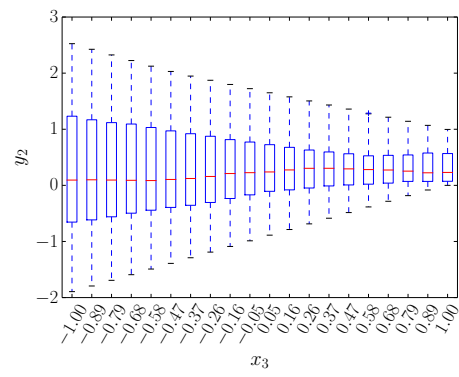

(f) Interacting (Heteroscedastic)

Figure 1: Illustrative Functions: the distributions $p\left(y \mid x_{i}\right)$ of equations 23 and 24 .

Table 4: Variances of $y_{1}$ and $y_{2}$ at $x_{1}=1,3$

\begin{tabular}{ccc}
\hline$x_{1}$ & $\underset{x_{-1}}{V}\left(y_{1} \mid x_{1}\right)$ & $\underset{x_{-1}}{V}\left(y_{2} \mid x_{1}\right)$ \\
\hline 1 & 1 & 1 \\
3 & 1 & 9 \\
\hline
\end{tabular}

contribution of $x_{1}$ renders different $\underset{x_{-1}}{V}\left(y_{2} \mid x_{1}\right)$ between $x_{1}=1$ and $x_{1}=3$ as observed in Table 4 . With this heteroscedasticity, we say that $x_{1}$ and $x_{2}$ are interacting.

Thus, from Table 4 we compute

$$
\begin{aligned}
& \underset{x_{1}}{V}\left[\underset{x_{-1}}{V}\left(y_{1} \mid x_{1}\right)\right]=0 \\
& \underset{x_{1}}{V}\left[\underset{x_{-1}}{V}\left(y_{2} \mid x_{1}\right)\right]=16 .
\end{aligned}
$$

The same procedure can be repeated for $x_{2}$.

The column change at $x_{i}$ leaves other columns $x_{-i}$ unchanged (as observed in Table 2 and Table 3), thus if $x_{i}$ contribution to an output $y$ is additive, $V\left(y \mid x_{i}\right)$ remains unchanged throughout the different values in $x_{i}$. This suggests that for screening purposes, we can let the Monte Carlo samples very low, and in the example above we had $m=2$, the minimum to compute a variance. Of course, at such low number, we cannot hope to have an accurate $V\left(y \mid x_{i}\right)$ because the distribution $p\left(y \mid x_{i}\right)$ will not be represented adequately. However, if $x_{i}$ is non-interacting,

$$
\underset{x_{i}}{V}\left[\underset{x_{-i}}{V}\left(y \mid x_{i}\right)\right]
$$

should give zero to an arithmetic precision. As $m$ is increased, $V\left(y \mid x_{i}\right)$ becomes accurate and a quantitative ordering of interaction among different input variables becomes possible. Furthermore, this "variance of variance" is never negative due to its sum-of-squares computations.

The normalizing factor $1 / V(y)$ in equation (16) is rather arbitrary, one could have equally applied, for example,

$$
\frac{1}{\sum_{i=1}^{n} \underset{x_{i}}{V}\left[{ }_{x_{-i}}^{V}\left(y \mid x_{i}\right)\right]}
$$

to mimic probability measures. However, in our opinion, this would not add much to the intuitive appeal and we have opted for the simpler expression.

A physical interpretation of $I_{i}$ is as follows. Consider a Dirac delta function $\delta\left(x_{i}-a\right)$, which is a distribution of $x_{i}$ and has a probability mass of 1 at $x_{i}=a$ and zero anywhere else. The interaction index $I_{i}$ shows the sensitivity (variance) of $\underset{x_{-i}}{V}\left(y \mid x_{i}\right)$ with respect to $a$ 
when the original uniform distribution of $x_{i}$ is replaced by $\delta\left(x_{i}-a\right), a \in \Omega_{i}$. Here, $\Omega_{i}$ is simply a real closed interval between upper and lower bounds of $x_{i}$. If you need to know which input variable $x_{i}$, if made deterministic, would make the uncertainty in the output $y$ most different depending on its input value $a$, the indices can be useful.

One may wonder, given a dataset of unknown input distributions, if $I_{i}=0$ would imply that the covariance between $x_{i}$ and another $x_{j}$ with $j \neq i$ would also be zero. However, this is not necessarily the case. An easy counter example is letting $x_{2} \sim N\left(x_{1}, 1\right)$ in equation (19). That is, $x_{2}$ are drawn from a normal distribution with mean $x_{1}$ with a constant standard deviation $\sigma=1$. In this case, $\operatorname{Cov}\left(x_{1}, x_{2}\right)>0$ but $I_{i}=0$.

\section{Comparison}

It is also possible to evaluate interaction via the total effect indices and first order Sobol indices, $S_{T i}-S_{i}$. However, there are some important differences between $I_{i}$ and $S_{T i}-S_{i}$.

First, $S_{T i}-S_{i}$ gives the variance in expected values of output $y$ due to $x_{i}$ that are not due to the first-order terms of equation (3) but by the second-order terms of equation (4) or higher. So it is a combined effect of more than one input variables to obtain the average output, for example $E\left(y \mid x_{i}, x_{j}\right)$. Fixing $x_{i}$ and $x_{j}$ with different combinations of values generates $V\left[E\left(y \mid x_{i}, x_{j}\right)\right]$ to obtain $S_{i j}$. In contrast, $I_{i}$ is a "first-order" index. Fixing $x_{i}$ at various values generates various $V\left(y \mid x_{i}\right)$ to obtain $V\left[V\left(y \mid x_{i}\right)\right]$. For example, consider again Figure 1. From Figure $1 \mathrm{e}$ and Figure 1f one would guess $I_{2}<I_{3}$ because by visual inspection, the difference in variance given a specific value in $x_{i}$ is greater for $V\left(y_{2} \mid x_{3}\right)$ than $V\left(y_{2} \mid x_{2}\right) . S_{T i}-S_{i}$ does not give information about the relative importance between $x_{i}$ and $x_{j}$ in driving the variance of $y_{2}$. On the other hand, $I_{i}$ does not distinguish the additive effect and the interaction effect of a single input variable. If $x_{i}$ interacts, it does not by itself give any indication of the elementary effect that it may have as in $S_{i}$.

Second, the detection of non-interaction $I_{i}=0$ is not sensitive to the accuracy of $V\left(y \mid x_{i}\right)$. As long as $V\left(y \mid x_{i}\right)$ is computed with the same samples in $x_{-i}, V\left(y \mid x_{i}\right)$ remains constant throughout different values of $x_{i}$ if $x_{i}$ gives only an additive effect to the output. In other words, if we have a matrix with $m$ rows of Monte Carlo samples with $n$ columns corresponding to the number of input variables and replace column $i$ with a value for $x_{i}$, and compute the corresponding outputs to obtain $V\left(y \mid x_{i}\right)$, this variance is identical regardless of the value of $x_{i}$ when variable $x_{i}$ is not interacting with other input variables. Thus, $I_{i}$ should show zero to arithmetic precision if $x_{i}$ does not interact with other variables. If the typical output variance $V\left(y \mid x_{i}\right)$ is in the order of $10^{0}$, non-interaction would typically produce $V\left[V\left(y \mid x_{i}\right)\right] \simeq 10^{-16}$ when computations are done in double precision. On the other hand, $S_{T i}-S_{i}$ is subject to the Monte Carlo integration inaccuracy.

For first order sensitivity calculations, $m$ is typically in the order of 1000 or above and $l$ is typically 50 or above in our experience. However, for screening purposes $I_{i}$ can be computed with $m$ and $l$ as low as 2 giving $4 n+2$ function evaluations. We need two samples to compute the output variances at two different values of an input variable and check that the variances of the two output values do not change with respect to the values of the input variable.

Lastly, for quantitative uses, the computation of $I_{i}$ does not require any further function evaluation (i.e., computation of response $y$ ) beyond what is required for the computation of first order Sobol indices $S_{i}$ in bruteforce approach. Computing $S_{T i}$ in brute-force approach is often infeasible (requiring computation of up to $n-1$ order Sobol indices), but efficient ways exist [10]. Furthermore, surrogate modeling techniques that facilitate the acquisition of $S_{i}$ and $S_{T i}$ exist such as using Polynomial Chaos Expansions $[14,15,16]$. We expect that there are shortcuts to economize the computation of $I_{i}$ as well. This is an open research topic and future work.

\section{Examples}

In this section, five functions will be analyzed using the proposed interaction indices and the conventional method of using the difference between total effects and first order sensitivity indices of Sobol'. The inputs will be assumed to be random variables with uniform distributions between upper and lower bounds. The numerical results and plots were obtained using a 32-bit version of Python 2.7.5, Numpy 1.8.0, Scipy 0.13.2, and Matplotlib 1.3.1.

The $S_{T i}-S_{i}$ is calculated using the methods described in Appendix A. The $I_{i}$ is calculated using the "bruteforce" approach. In both $S_{T i}-S_{i}$ and $I_{i}$, uniform random sampling is used for Monte Carlo integrations.

\subsection{Illustrative Functions}

Consider the following simple example.

$$
\begin{aligned}
& y_{1}=x_{1}+2 x_{2}+4 x_{3} \\
& y_{2}=x_{1}^{2}-x_{2}+x_{2} x_{3}
\end{aligned}
$$


where $-1<x_{1}, x_{2}, x_{3} \leq 1$. Figure 1 shows marginal distributions as box plots. For these plots, Monte Carlo sampling was performed using the brute-force approach with $m=200$ and $l=20$.

The interaction indices are shown in Table 5. The

Table 5: First order interaction indices for the Illustrative Functions

\begin{tabular}{|l|ll|}
\hline & $y_{1}$ & $y_{2}$ \\
\hline$I_{1}^{2}$ & 0.000 & 0.000 \\
$I_{2}^{2}$ & 0.000 & 0.036 \\
$I_{3}^{2}$ & 0.000 & 0.573 \\
\hline
\end{tabular}

zero entries in Table 5 indicate that corresponding variables do not interact with other variables. For $y_{2}, x_{1}$ is non-interacting, but $x_{2}$ and $x_{3}$ are interacting.

Table 6 shows the result of calculating $S_{T i}-S_{i}$ with $m=30200$. The column for $y_{1}$ and the entry for $x_{1}$ under the column for $y_{2}$ show zeros if we round to the second decimal place. For the $y_{2}$ column, the entry for $x_{2}$ and

Table 6: $S_{T i}-S_{i}$ for the Illustrative Functions

\begin{tabular}{|l|ll|}
\hline & $y_{1}$ & $y_{2}$ \\
\hline$S_{T 1}-S_{1}$ & 0.00 & 0.00 \\
$S_{T 2}-S_{2}$ & 0.00 & 0.21 \\
$S_{T 3}-S_{3}$ & 0.00 & 0.21 \\
\hline
\end{tabular}

$x_{3}$ show the interaction. Equations (25) to (27) show the expressions of $S_{T i}-S_{i}$ for $y_{2}$. The reason that

$$
S_{T 2}-S_{2}=S_{T 3}-S_{3}
$$

in Table 6 can be understood from equations (26) and (27).

$$
\begin{aligned}
& S_{T 1}-S_{1}=S_{12}+S_{13}+S_{123}=0, \\
& S_{T 2}-S_{2}=S_{12}+S_{23}+S_{123}=S_{23}, \\
& S_{T 3}-S_{3}=S_{13}+S_{23}+S_{123}=S_{23},
\end{aligned}
$$

because $S_{12}=S_{13}=S_{123}=0$. The difference between Table 5 and Table 6 illustrates the difference between the two methods of detecting interactions and noninteractions. The reason for $I_{2}<I_{3}$ in Table 5 can be understood by factoring equation (24) as in equation (28),

$$
y_{2}=x_{1}^{2}+x_{2} \cdot\left(-1+x_{3}\right) .
$$

For the given upper and lower bounds of $x_{2}$ and $x_{3}$, we have $-2<-1+x_{3} \leq 0$ and $-1<x_{2} \leq 1$. Thus, if we sample $x_{2}$ and $x_{3}$ uniformly between -1 and 1 , we have the following. If we let $x_{2}=1$ or -1 , then we get the largest $V\left(y_{2} \mid x_{2}\right)$ with

$$
V\left[x_{2} \cdot\left(-1+x_{3}\right) \mid x_{2}= \pm 1\right]=\frac{1}{3} \text {. }
$$

On the other hand, if we let $x_{3}=-1$, then

$$
V\left[x_{2} \cdot\left(-1+x_{3}\right) \mid x_{3}=-1\right]=\frac{4}{3},
$$

and $V\left(y_{2} \mid x_{3}\right)$ is largest. Furthermore,

$$
\begin{aligned}
& V\left[x_{2} \cdot\left(-1+x_{3}\right) \mid x_{2}=0\right]=0, \\
& V\left[x_{2} \cdot\left(-1+x_{3}\right) \mid x_{3}=1\right]=0 .
\end{aligned}
$$

Thus,

$$
\frac{I_{3}^{2}}{I_{2}^{2}}=\frac{V\left[V\left(y_{2} \mid x_{3}\right)\right]}{V\left[V\left(y_{2} \mid x_{2}\right)\right]}=\frac{4^{2}}{1^{2}}=16,
$$

which confirms Table 5.

\subsection{Ishigami Function}

Ishigami function $[17,18]$ is a three-variable function with an interaction between two of its input variables.

$$
y_{1}=\sin x_{1}+a \sin ^{2} x_{2}+b x_{3}^{4} \sin x_{1}
$$

where $-\pi<x_{1}, x_{2}, x_{3}<\pi$. In this paper, we set $a=7$ and $b=0.1$.

Figure 2 confirms visually that $x_{1}$ and $x_{3}$ are the interacting variables with their heteroscedastic behaviors. Figure 3 shows the distribution of values of $S_{T i}-S_{i}$ and $I_{i}$ of 20 independent runs for the function (Appendix B). As stated before, $S_{T i}-S_{i}$ is calculated with $m \times(n+2)$, and $I_{i}$ with $m \times(n \times l+1)$ function evaluations. Three different settings are tried out with different values for $m$ and $n$. The difference in the values of $m$ between the two methods is to make two methods perform about the same number of function evaluations.

As can be observed in Figure 3 a to Figure $3 \mathrm{c}, S_{T i}-S_{i}$ loses accuracy as $m$ becomes smaller. At a low number of $m$ such as in Figure 3c, it would be impossible to detect interactions happening between $x_{1}$ and $x_{3}$ or the additive effect of $x_{2}$. On the other hand, for $I_{i}$ as in Figure $3 \mathrm{~d}$ to Figure $3 \mathrm{f}$, even though their values become less accurate as $m$ is decreased, the non-interaction of $x_{2}$ can clearly be detected by setting a threshold $\varepsilon$, for example $\varepsilon=10^{-9}$. We also see the relative importance of $x_{3}$ compared to $x_{2}$ in Figure $3 \mathrm{~d}$ and Figure $3 \mathrm{e}$ in terms of variance of distribution $p\left(y \mid x_{i}\right)$ or $\underset{x_{i}}{V}\left[V\left(y \mid x_{-i}\right)\right.$.

\subsection{G-Function}

Sobol's G-function $[19,20,10]$ is a test function for which global sensitivity can be controlled via its parameters. We use an eight-dimensional setting described in $[20]$.

$$
y_{1}=\prod_{i=1}^{n} g_{i}\left(x_{i}\right)
$$




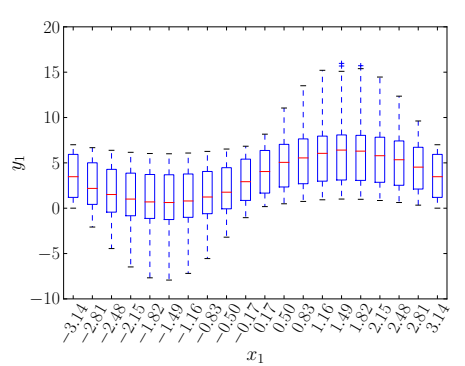

(a) Interacting (Heteroscedastic)

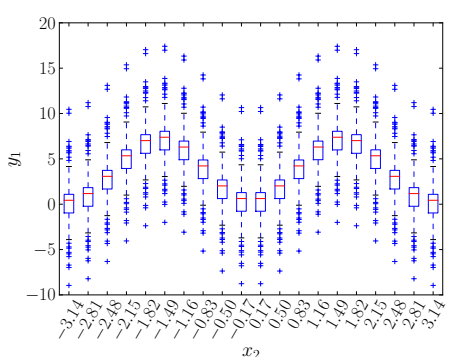

(b) Additive (Homoscedastic)

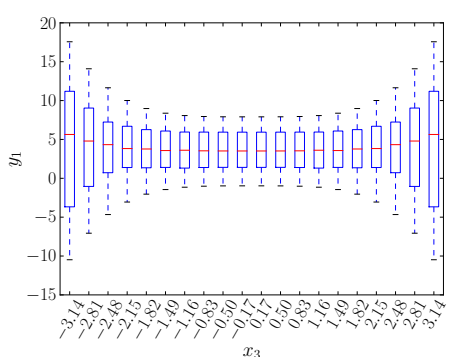

(c) Interacting (Heteroscedastic)

Figure 2: Ishigami Function: distributions of $p\left(y \mid x_{i}\right)$ or the marginal views

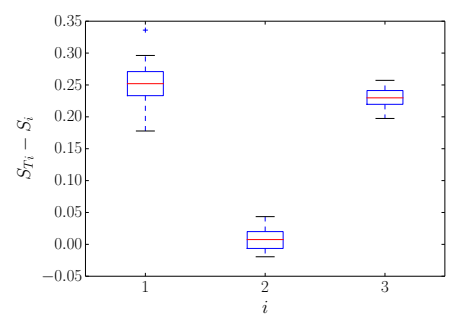

(a) $m=2440$

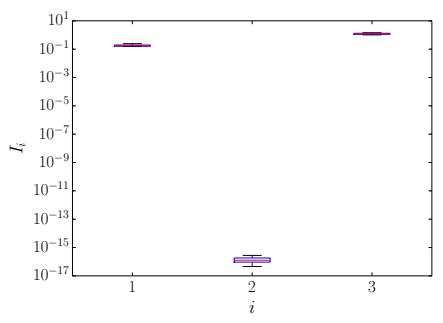

(d) $m=200, l=20$

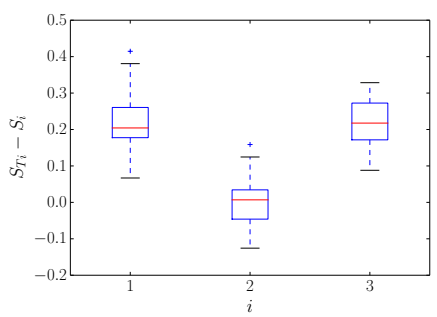

(b) $m=244$

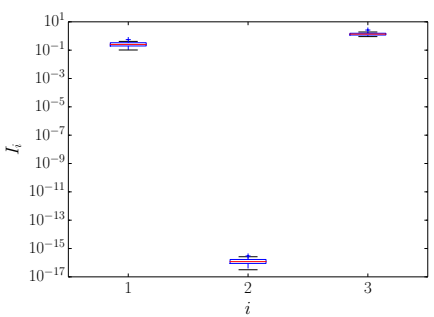

(e) $m=20, l=20$

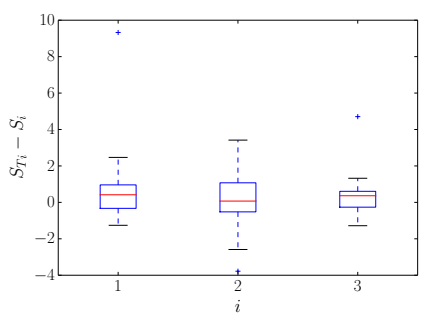

(c) $m=3$

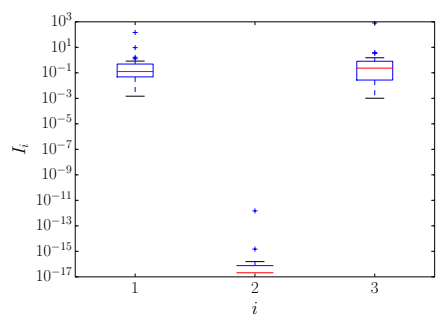

(f) $m=2, l=2$

Figure 3: Ishigami Function: box plots show the distribution of indices values of 20 runs. 
where

$$
g_{i}\left(x_{i}\right)=\frac{\left|4 x_{i}-2\right|+a_{i}}{1+a_{i}}, 0 \leq x_{i}<1,
$$

with $n=8$, and $\left\{a_{i}\right\}=\{0,1,4.5,9,99,99,99,99\}$. For $x_{i}$ with $a_{i}=0$, the variable is very important. On the other hand, if $a_{i}=99, x_{i}$ 's effect is negligible, but still interacting with other variables. In Figure 4, we see that $I_{i}$ cannot reliably quantify relative importance of each variable when $m$ is very low as observed in Figure $4 \mathrm{f}$. However, we can still see that all the variables from $x_{1}$ to $x_{8}$ that they are all interacting since $I_{i}>10^{-6} \gg 10^{-16}$. On the other hand, Figure $4 \mathrm{c}$ shows that $S_{T i}-S_{i}$ is too unreliable at this number of samples.

\subsection{Rosenbrock - Sphere Function}

This function is simply a combination of two famous functions. We set the first five dimensions to be the inputs to the Rosenbrock function and the remaining five to be the inputs to the sphere function.

$$
\begin{aligned}
y_{1} & =\sum_{i=1}^{\lfloor n / 2\rfloor-1}\left[100\left(x_{i+1}-x_{i}^{2}\right)^{2}+\left(1-x_{i}\right)^{2}\right] \\
& +1000 \sum_{i=\lfloor n / 2\rfloor+1}^{n} x_{i}^{2}
\end{aligned}
$$

where $-10 \leq x_{i} \leq 10$, and $n=10$.

For this function, $x_{6}$ to $x_{10}$ have no interactions while the first five variables have interactions. We see in Figure 5a that $S_{T i}-S_{i}$ cannot provide a reliable quantitative information about interaction, even at fairly high number of function evaluations: $33500 \times(10+2)=402000$. The small interaction values make it difficult to be detected under Monte Carlo integration accuracy. On the other hand, Figure $5 \mathrm{~d}$ to Figure $5 \mathrm{f}$ show that, for $I_{i}$, noninteracting variables remained discernible, even if the accuracy of indices deteriorated (as evidenced by the increase in the spread of box plots).

\subsection{Artery Simulation}

In this example, we investigate an application of our method to a physics-based problem of parameter identification. The simulation code we use was developed by Degroote et al. [21]. This code has recently been used as an example problem for Gradient Enhanced Kriging [22] since the function exposes the gradient as well as the objective value. For our purpose, we will ignore the gradients and treat it as a scalar function with vector input consisting of the parameters we want to identify.
The code simulates the hemodynamics of the arterial system as one-dimensional fluid-structure interaction problem. Figure 6 shows a schematic of an axisymmetric model of the artery system along with its boundary conditions. The modeled blood flow in an artery is the unsteady flow of an incompressible, inviscid fluid, in a straight, flexible tube. The flow rate at the inlet is prescribed as a function of time. The outlet has velocity extrapolated using the velocities of the last two segments and relates to the output pressure using the Windkessel model [23, 24]. The Windkessel model represents the hemodynamics of the circulation downstream. Its dynamics is expressed using an electrical circuit analogy. A so-called generalized string model is applied to the structure. This is a linear elasticity theory for a thin cylindrical tube with membrane deformations $[25,26]$.

In this exercise, the inputs $x_{i}$ are the modulus of elasticities of the artery at $n-1$ segments and the value of capacitance of the downstream boundary condition, totaling $n$ input variables. We let $n=20$. The output $y$ is the sum of squared error between the simulated values and reference values (a priori obtained by the same simulation code in this example) of the radii of the artery at these segments. The sum $y$ is over all time steps and all artery segments. This sum $y$ is normalized so that it will not exceed 1. Exact match in time histories of radii between the given reference values and the simulation would give zero in the output. In a real situation, the reference values of radii would come from non-invasive measurements such as from ultrasound imagery.

Thus, the function we are analyzing can be expressed as

$$
y=f(\mathbf{T}, \mathbf{x}),
$$

where $\mathbf{T}$ is the matrix containing reference values of the radii of all $n-1$ segments for all timesteps, and $\mathbf{x}$ is the vector containing input variables $x_{i}$. The $\mathbf{T}$ is given, and we sample $\mathbf{x}$ to see whether its elements interact to obtain the output $y$. We pretend that we do not know the input $\mathbf{x}$ that generated the reference time histories of the radii $\mathbf{T}$, but have a rough idea to form the domain of the function. Specifically we create a $\pm 50 \%$ interval around nominal values $E_{0}$ and $C_{0}$ (Table 7). We investigate how the input variables interact to produce (the sum of errors in) the output. The inputs $x_{i}$ are scaled to take values between -1 and 1 such as the following.

$$
\begin{aligned}
E_{i} & =E_{0}\left(1+\frac{1}{2} x_{i}\right), i \in\{1, \cdots, n-1\}, \\
C & =C_{0}\left(1+\frac{1}{2} x_{n}\right)
\end{aligned}
$$

where $E_{i}$ is the modulus of elasticity of $n-1$ artery 


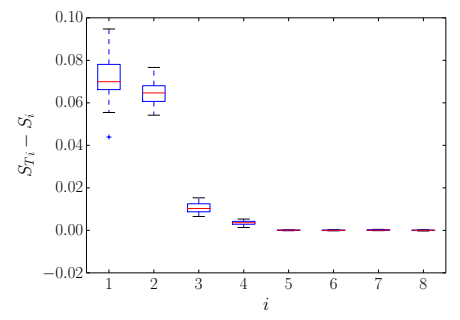

(a) $m=32200$

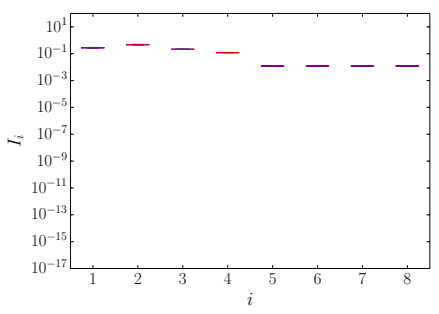

(d) $m=2000, l=20$

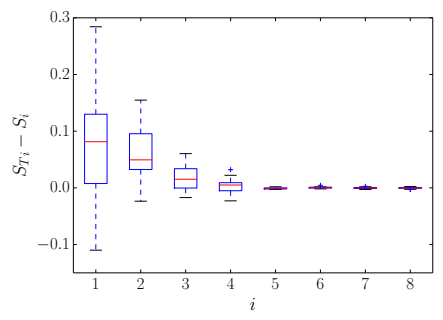

(b) $m=322$

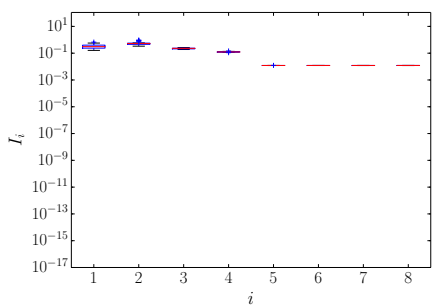

(e) $m=20, l=20$

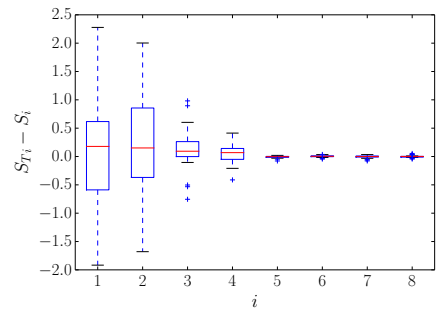

(c) $m=4$

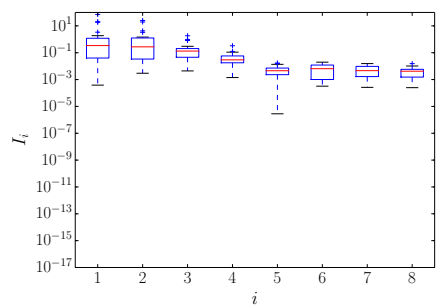

(f) $m=2, l=2$

Figure 4: G Function: box plots show the distribution of indices values of 20 runs.

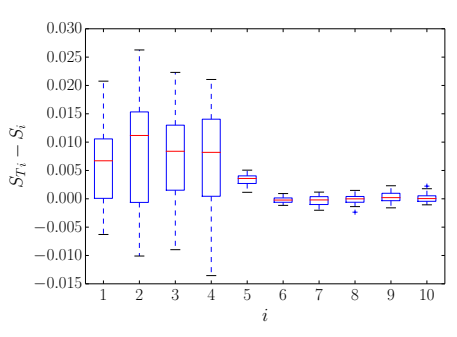

(a) $m=33500$

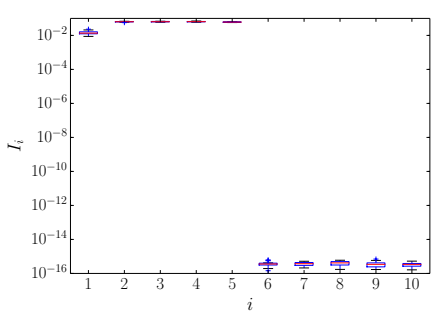

(d) $m=2000, l=20$

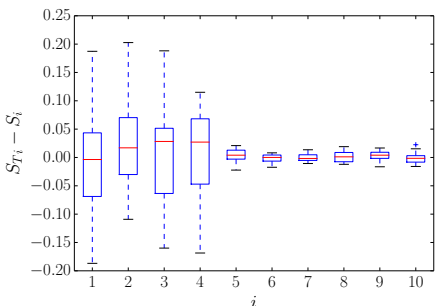

(b) $m=335$

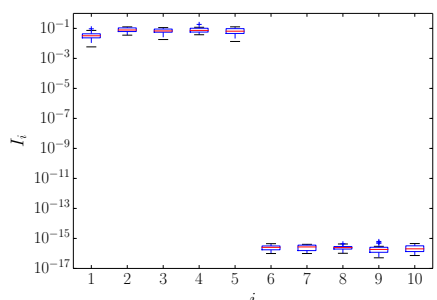

(e) $m=20, l=20$

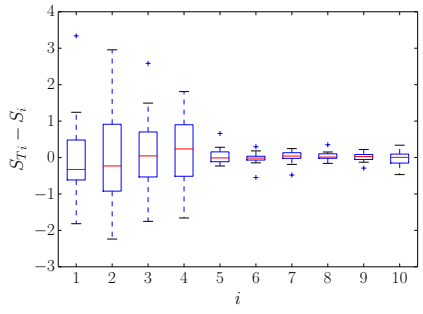

(c) $m=4$

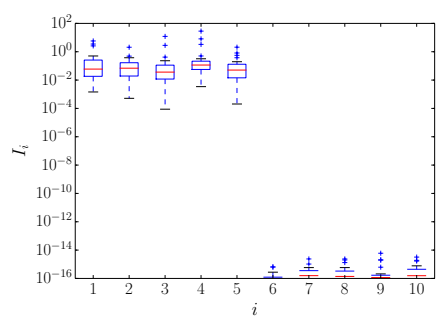

(f) $m=2, l=2$

Figure 5: Rosenbrock - Sphere Function: box plots show the distribution of indices values of 20 runs. 
segments and $C$ is the capacitance in the Windkessel model representing the compliance of the arterial system. Table 7 show the parameter values used in the artery model.

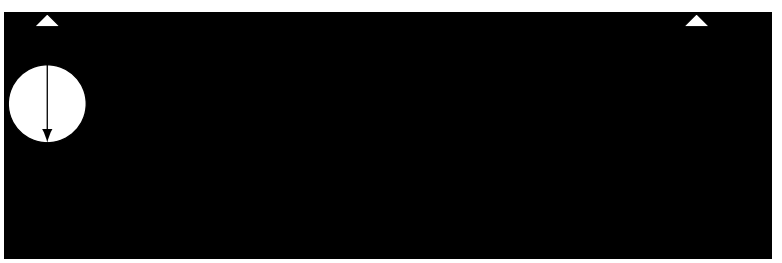

Figure 6: The diagram of an artery model with blood flowing in from left with prescribed time-dependent velocities and flowing out at the right with the Windkessel model pressure. The segments (eight in the figure), the radius $r$, the wall thickness $h$ and the length $l$ are shown. The prescribed inlet flow rate is given by $u_{0}(t)=0.23+0.21 \sin \left(2 \pi \frac{t}{t_{b}}\right)+0.11 \cos \left(4 \pi\left(\frac{t}{t_{b}}-0.2\right)\right)+$ $0.07 \cos \left(6 \pi\left(\frac{t}{t_{b}}-0.2\right)\right)$, where $t_{b}$ is the pulse period.

Table 7: Parameter values used in the artery model

\begin{tabular}{clll}
\hline$r_{0}$ & $3 \times 10^{-3} \mathrm{~m}$ & $E_{0}$ & $4 \times 10^{5} \mathrm{~Pa}$ \\
$h$ & $3 \times 10^{-4} \mathrm{~m}$ & $C_{0}$ & $6.35 \times 10^{-10} \mathrm{~m}^{3} / \mathrm{Pa}$ \\
$l$ & $0.126 \mathrm{~m}$ & $R_{d}$ & $1.768 \times 10^{9} \mathrm{~Pa} \mathrm{~s} / \mathrm{m}^{3}$ \\
$t_{b}$ & $1 \mathrm{~s}$ & $R_{p}$ & $2.834 \times 10^{8} \mathrm{~Pa} \mathrm{~s} / \mathrm{m}^{3}$ \\
\hline
\end{tabular}

The results of computations of $S_{T i}-S_{i}$ and $I_{i}$ are shown in Figure 7. The computation time to obtain Figure 7a and Figure 7c combined was 233412 seconds or approximately 65 hours on a laptop computer with Intel Core2 Duo 2.8 GHz CPU and 4.0 GB RAM. For the computation of Figure $7 \mathrm{~b}$ and Figure $7 d$ combined, the elapsed time was 2552 seconds or about 43 minutes on the same computer.

For this problem, one would expect that all parameters have interactions. However, $S_{T i}-S_{i}$ values in Figure $7 \mathrm{a}$ and Figure $7 \mathrm{~b}$ were not consistent enough throughout the 20 runs to show the interactions of elasticities of the arteries, with many of the indices showing below zero values. On the other hand, we obtained $I_{i}>10^{-6} \gg 10^{-16}$ in Figure $7 \mathrm{c}$ and Figure $7 \mathrm{~d}$ and one would be able to confirm the interactions.

The capacitance or the compliance parameter C (at $i=20$ ) gave markedly higher values for both $S_{T i}-S_{i}$ and $I_{i}$ in Figure 7a and Figure 7c. This can be understood from the fact that the parameter is part of the downstream boundary condition affecting the time histories of radii of all the 19 upstream segments. In either case, the spread of the boxes indicates that the numbers of $m$ in Figure 7a and Figure 7c were not large enough to show the relative importance of interactions among the elasticities of the artery segments. The same holds for smaller $m$. With Figure $7 \mathrm{~b}$ and Figure $7 \mathrm{~d}$, neither $S_{T i}-S_{i}$ nor $I_{i}$ were able to capture reliably the salient importance of the capacitance parameter $(i=20)$.

\section{Discussion and Outlook}

As can be seen in Figure 3 and Figure $4, I_{i}$ and $S_{T i}-S_{i}$ do not necessarily give a consistent ranking of importance (i.e., importance ordering of interacting input variables differ between the two methods). This is due to the fact that the indices evaluate the interaction in different ways as explained in Section 6 and 7. In $I_{i}$, it quantifies how sensitive the output variance is if we fix $x_{i}$ to a value "a" rather than another value "b", for example. On the other hand, $S_{T i}-S_{i}$ quantifies the uncertainty in output remaining after subtracting the main effect uncertainty. Therefore, if the parameter $x_{i}$ is uncertain by nature the more relevant interaction measure would be $S_{T i}-S_{i}$. However, if we can turn $x_{i}$ into a deterministic variable and we can choose its value, $I_{i}$ can give an appropriate measure. The implication of this difference merits further studying.

In practical situations in which the calculation of $y$ given an input vector $\mathbf{x}$ is expensive, the computation of variance based sensitivity indices and quantitative interaction analysis of input variables may be prohibitive due to the number of model evaluations needed to do the Monte Carlo integration. In such cases, fitting surrogate models to the dataset computed by the original model may be useful. Surrogate models [27, 28] are approximations to the original function and are much cheaper to compute than the original model. It is usually fitted to a finite number of input-output data obtained from the original model (usually a complex simulation model). Kriging and Radial Basis Functions are some of the popular surrogate models performing interpolations.

There are also regression methods based on HDMR $[29,30,31]$. The basis functions in these are polynomials. The representations are usually truncated at second order or so, thus ignoring higher order terms and interactions. Let us denote the output produced by the surrogate model as $\hat{y}$. We can compute the indices based on $\hat{y}$ 's. However, information about interactions may be inaccurate due to the approximate nature of the surrogate model. Furthermore, interpolating surrogate models are usually not very scalable to high-dimensional problems. Our proposed method could be applied to the high-fidelity model for screening purposes, and potentially for determining what interaction terms to include in HDMR based regressions. The same method could then be applied for quantitative purposes in the reduced 


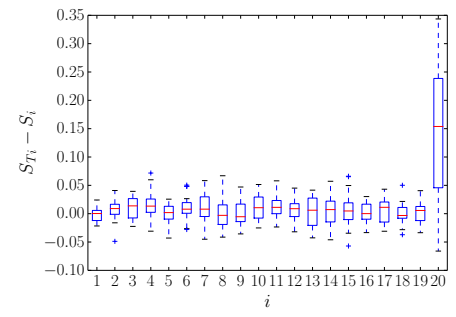

(a) $m=365$

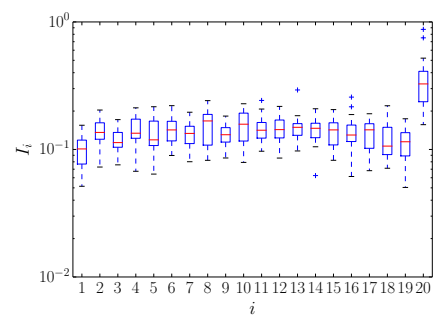

(c) $m=20, l=20$

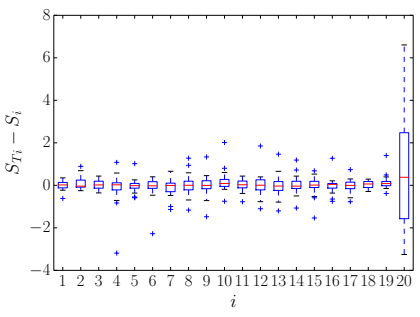

(b) $m=4$

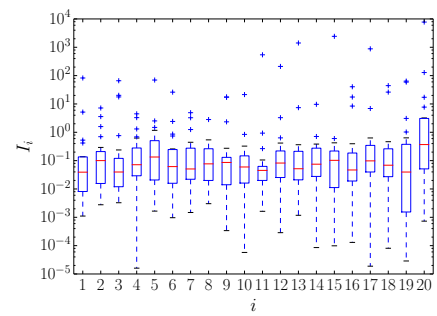

(d) $m=2, l=2$

Figure 7: Artery fluid-structure simulation for model calibration of 19 elasticity parameters $(i \in\{1, \cdots, 19\})$ and a downstream compliance parameter (the capacitance, $i=20$ ): box plots show the distribution of index values of 20 runs.

problem (possibly on a surrogate model). Further research would be beneficial to see the actual merit of this approach.

\section{Conclusion}

The interaction index exposes each variable's importance in influencing the variance in the output through interaction. Its accuracy does not directly depend on the accuracy of the Monte Carlo integration, but on the change in the sample marginal distribution or heteroscedasticity. The examples showed its robustness in detecting and quantifying interactions among input variables. This is expected to be useful in (robust) optimization and surrogate modeling typical in engineering analysis and design. Further application to industrial problems is needed to understand the effectiveness of the proposed index. Also, further research would be useful to exploit the concept described in this paper to develop a surrogate model assisted optimization algorithm that is scalable to high-dimensional problems.

\section{Acknowledgments}

Keiichi Ito has been funded by the Institute for the Promotion of Innovation through Science and Technology (IWT) through the Baekeland Mandate pro- gram. Ivo Couckuyt is a post-doctoral research fellow of FWO-Vlaanderen. This research has also been funded by the Interuniversity Attraction Poles Programme BESTCOM initiated by the Belgian Science Policy Office.

\section{Appendix A. Monte Carlo Estimation of Indices}

In the following, we give the Monte Carlo estimation of the indices. The method for $I_{i}$ follows the so called "brute-force" method that would entail a double loop in a computer code. We consider an $n$-dimensional unit hypercube domain for notational brevity. Let $\mathbf{A}$ and $\mathbf{B}$ be two matrices with uniform random value elements between 0 and 1 . The two matrices have the size of $m$ rows and $n$ columns. Let $j$ and $i$ be row and column indices, respectively. The notation $\mathbf{A}_{\mathbf{B}}{ }^{(i)}$ means that all columns are from $\mathbf{A}$ except column $i$ which is from $\mathbf{B}$. For total variance of output $y$, we can use

$$
D \simeq \frac{1}{2 m-1} \sum_{j=1}^{2 m} f(\mathbf{C})_{j}^{2}-f_{0_{C}}^{2}
$$

where $\mathbf{C}$ is the concatenated matrix of $\mathbf{A}$ and $\mathbf{B}$ with $2 m$ rows and $n$ columns and $f_{0_{C}}$ is the mean of $f(\mathbf{C})_{j}$, or 
alternatively:

$$
\begin{aligned}
D_{A} \simeq & \frac{1}{m-1} \sum_{j=1}^{m} f(\mathbf{A})_{j}^{2}-f_{0_{A}}^{2} \\
D_{B} \simeq & \frac{1}{m-1} \sum_{j=1}^{m} f(\mathbf{B})_{j}^{2}-f_{0_{B}}^{2} \\
D_{A B} \simeq & \frac{1}{2 m-1} \sum_{j=1}^{2 m} f(\mathbf{C})_{j}^{2} \\
& -\frac{1}{m-1} \sum_{j=1}^{m} f(\mathbf{A})_{j} f(\mathbf{B})_{j}
\end{aligned}
$$

where $f_{0_{A}}$ and $f_{0_{B}}$ are the mean of $f(\mathbf{A})_{j}$ and $f(\mathbf{B})_{j}$, respectively. In our calculation of $S_{i}$ and $S_{T i}$, we used equation (A.1). The best-practice [10] recommends to compute the $D_{i}$ in equation (13) as in the following.

$$
D_{i} \simeq \frac{1}{m-1} \sum_{j=1}^{m} f(\mathbf{B})_{j}\left(f\left(\mathbf{A}_{\mathbf{B}}{ }^{(i)}\right)_{j}-f(\mathbf{A})_{j}\right)
$$

Thus, first-order sensitivity index is

$$
S_{i}=\frac{D_{i}}{D}
$$

For total effects,

$$
S_{T i} \simeq \frac{1}{2 D(m-1)} \sum_{j=1}^{m}\left(f(\mathbf{A})_{j}-f\left(\mathbf{A}_{\mathbf{B}}{ }^{(i)}\right)_{j}\right)^{2} .
$$

Let $k$ be the index of $l$ levels of $x_{i}$. We designate $k$ th level of $x_{i}$ as $x_{i k}$ and matrix $\mathbf{A}$ with $i$ th column replaced by element $x_{i k}$ as $\mathbf{A}_{x_{i k}}^{(i)}$. Then, interaction indices can be computed from

$$
\begin{aligned}
\underset{x_{i}}{V}\left[{ }_{x_{-i}}\left(y \mid x_{i}\right)\right] \simeq & \frac{1}{l-1} \sum_{k=1}^{l}\left(\frac{1}{m-1} \sum_{j=1}^{m} f\left(\mathbf{A}_{x_{i k}}^{(i)}\right)_{j}^{2}-f_{o_{A}}^{2}\right)^{2} \\
& -\mu_{V\left(f\left(A_{x_{i}}^{(i)}\right)\right)}^{2}
\end{aligned}
$$

where $\mu_{V\left(f\left(A_{x_{i}}^{(i)}\right)\right)}$ is the average variance of $f\left(A_{x_{i}}^{(i)}\right)$ when $x_{i}$ is varied through $l$ levels. Then, $I_{i}^{2}$ can be obtained by dividing the result from equation (A.7) by $D_{A}^{2}$.

\section{Appendix B. Sample Size for Box Plots}

Our objective in the box plots was not to support any significance tests, but to show qualitatively the problems that may arise. The number of independent runs was not determined on statistically rigorous grounds, but by the desire to keep the computational costs to an easily manageable level. There seems to be no theoretical foundation of how large the sample size for box plots should be, except that it should be at least 5 [32]. There is no universally agreed method of computing the box boundaries. We employ the Tukey-Style box plots as implemented in the Python module Matplotlib, in which the whiskers extend up to 1.5 times the Inter Quartile Range. The choice of 20 independent runs of $S_{T i}-S_{i}$ and $I_{i}$ estimations to generate the box plots in Figure 3, 4, 5, and 7 was determined taking into account the guidelines given by Krzywinski and Altman [32] and Minitab Express Support [33].

\section{References}

[1] T. Homma, A. Saltelli, Importance Measures in Global Sensitivity Analysis of Nonlinear Models, Reliability Engineering and System Safety 52 (1996) $1-17$.

[2] I. M. Sobol', Global sensitivity indices for nonlinear mathematical models and their Monte Carlo estimates, Mathematics and Computers in Simulation 55 (2001) 271-280.

[3] A. Saltelli, Making Best Use of Model Evaluations to Compute Sensitivity Indices, Computer Physics Communications 145 (2002) 280 - 297.

[4] M. D. Morris, Factorial Sampling Plans for Preliminary Computational Experiments, Technometrics 33 (2) (1991) pp. 161-174, ISSN 00401706.

[5] F. Campolongo, A. Saltelli, J. Cariboni, From screening to quantitative sensitivity analysis. A unified approach, Computer Physics Communications 182 (4) (2011) 978 - 988, ISSN 0010-4655, doi: \bibinfo\{doi $\}$ http://dx.doi.org/10.1016/ j.cpc.2010.12.039\}.

[6] A. Saltelli, F. Campolongo, J. Cariboni, Screening important inputs in models with strong interaction properties, Reliability Engineering \& System Safety 94 (7) (2009) 1149 - 1155, ISSN 0951-8320, doi: \bibinfo\{doi\} \{http://dx.doi.org/10.1016/ j.ress.2008.10.007\}, special Issue on Sensitivity Analysis.

[7] S. Kucherenko, M. Rodriguez-Fernandez, C. Pantelides, N. Shah, Monte Carlo evaluation of derivative-based global sensitivity measures, Reliability Engineering \& System Safety 94 (7) (2009) 1135 - 1148, ISSN 0951-8320, doi: \bibinfo $\{$ doi $\}$ $\{$ http://dx.doi.org/10.1016/j.ress.2008.05.006\}, special Issue on Sensitivity Analysis.

[8] I. M. Sobol', Sensitivity Estimates for Nonlinear Mathematical Models, Mathematical Modeling and Computational Experiment 1 (4) (1993) 407 - 414.

[9] K. Chan, A. Saltelli, S. Tarantola, Sensitivity Analysis of Model Output: Variance-Based Methods Make the Difference, in: Proceedings of the 1997 Winter Simulation Conference, 261-268, 1997.

[10] A. Saltelli, P. Annoni, I. Azzini, F. Campolongo, M. Ratto, S. Tarantola, Variance based sensitivity analysis of model output. Design and estimator for the total sensitivity index, Computer Physics Communications 181 (2) (2010) 259 - 270, ISSN 0010-4655, doi: \bibinfo\{doi\} \{http://dx.doi.org/10.1016/ j.cpc.2009.09.018\}.

[11] T. A. Mara, S. Tarantola, P. Annoni, Non-parametric methods for global sensitivity analysis of model output with dependent inputs, Environmental Modelling \& Software 72 (2015) 173 - 183, ISSN 1364-8152, doi: \bibinfo\{doi $\}\{$ http: //dx.doi.org/10.1016/j.envsoft.2015.07.010\},
URL 
http://www.sciencedirect.com/science/article/ pii/S1364815215300153.

[12] T. A. Mara, S. Tarantola, Variance-based sensitivity indices for models with dependent inputs, Reliability Engineering \& System Safety 107 (2012) 115 - 121, ISSN 09518320, doi: $\backslash$ bibinfo $\{$ doi $\}$ \{http://dx.doi.org/10.1016/j.ress.2011. 08.008\}, URL http://www.sciencedirect.com/science/ article/pii/S0951832011001724, \{SAMO\} 2010.

[13] S. Kucherenko, S. Tarantola, P. Annoni, Estimation of global sensitivity indices for models with dependent variables, Computer Physics Communications 183 (4) (2012) 937 - 946, ISSN 0010-4655, doi: \bibinfo $\{$ doi $\}\{$ http://dx.doi.org/10.1016/ j.cpc.2011.12.020\}, URL http://www.sciencedirect.com/ science/article/pii/S0010465511004085.

[14] B. Sudret, Global sensitivity analysis using polynomial chaos expansions, Reliability Engineering \& System Safety 93 (7) (2008) 964 - 979, ISSN 0951-8320, doi: bibinfo\{doi\}\{http://dx.doi.org/10.1016/j.ress.2007.04.002\}, URL http://www.sciencedirect.com/science/article/ pii/S0951832007001329, bayesian Networks in Dependability.

[15] R. Pulch, E. J. W. ter Maten, F. Augustin, Sensitivity analysis and model order reduction for random linear dynamical systems, Mathematics and Computers in Simulation 111 (2015) 80 - 95, ISSN 0378-4754, doi: $\backslash$ bibinfo $\{$ doi $\}\{$ http://dx.doi.org/10.1016/ j.matcom.2015.01.003\}, URL http://www.sciencedirect. com/science/article/pii/S037847541500004X.

[16] G. T. Buzzard, D. Xiu, Variance-Based Global Sensitivity Analysis via Sparse-Grid Interpolation and Cubature, Communications in Computational Physics 9 (3) (2011) 542 - 567.

[17] T. Ishigami, T. Homma, An importance quantification technique in uncertainty analysis for computer models, in: Uncertainty Modeling and Analysis, 1990. Proceedings., First International Symposium on, IEEE, 398-403, doi: $\backslash$ bibinfo $\{$ doi $\}\{10$. 1109/ISUMA.1990.151285\}, 1990.

[18] I. Sobol', Y. Levitan, On the use of variance reducing multipliers in Monte Carlo computations of a global sensitivity index, Computer Physics Communications 117 (1999) 52 - 61, ISSN 0010-4655, doi: $\backslash$ bibinfo $\{$ doi $\}\{$ http://dx.doi.org/10.1016/ S0010-4655(98)00156-8\}.

[19] I. Sobol, S. Tarantola, D. Gatelli, S. Kucherenko, W. Mauntz, Estimating the approximation error when fixing unessential factors in global sensitivity analysis, Reliability Engineering \& System Safety 92 (7) (2007) 957-960, doi: \bibinfo $\{$ doi $\}\{$ http: //dx.doi.org/10.1016/j.ress.2006.07.001\}.

[20] D. Gatelli, S. Kucherenko, M. Ratto, S. Tarantola, Calculating first-order sensitivity measures: A benchmark of some recent methodologies, Reliability Engineering \& System Safety 94 (7) (2009) 1212 - 1219, ISSN 0951-8320, doi: $\backslash$ bibinfo $\{$ doi $\}\{$ http: //dx.doi.org/10.1016/j.ress.2008.03.028\}, special Issue on Sensitivity Analysis.

[21] J. Degroote, M. Hojjat, E. Stavropoulou, R. W uchner, K.U. Bletzinger, Partitioned solution of an unsteady adjoint for strongly coupled fluid-structure interactions and application to parameter identification of a one-dimensional problem, Structural and Multidisciplinary Optimization 47 (1) (2013) 77-94, ISSN 1615-147X, doi: $\backslash$ bibinfo $\{$ doi $\}\{10.1007 /$ s00158-012-0808-2\}, URL http://dx.doi.org/10.1007/ s00158-012-0808-2.

[22] S. Ulaganathan, I. Couckuyt, T. Dhaene, J. Degroote, E. Laermans, Performance study of gradient-enhanced Kriging, Engineering with Computers (2015) 1-20ISSN 0177-0667, doi: bibinfo $\{$ doi $\}\{10.1007 / \mathrm{s} 00366-015-0397-y\}$, URL http:// dx.doi.org/10.1007/s00366-015-0397-y.

[23] I. E. Vignon-Clementel, C. Figueroa, K. Jansen, C. Taylor, Out- flow boundary conditions for 3D simulations of non-periodic blood flow and pressure fields in deformable arteries, Computer methods in biomechanics and biomedical engineering 13 (5) (2010) 625-640.

[24] N. Westerhof, J.-W. Lankhaar, B. E. Westerhof, The arterial windkessel, Medical \& biological engineering \& computing 47 (2) (2009) 131-141.

[25] J.-F. Gerbeau, M. Vidrascu, A quasi-Newton algorithm based on a reduced model for fluid-structure interaction problems in blood flows, ESAIM: Mathematical Modelling and Numerical Analysis-Modélisation Mathématique et Analyse Numérique 37 (4) (2003) 631-647.

[26] A. Quarteroni, M. Tuveri, A. Veneziani, Computational vascular fluid dynamics: problems, models and methods, Computing and Visualization in Science 2 (4) (2000) 163-197.

[27] A. J. Keane, P. B. Nair, Computational Approaches for Aerospace Design: The Pursuit of Excellence, John Wiley \& Sons, 2005.

[28] D. Gorissen, K. Crombecq, I. Couckuyt, T. Dhaene, P. Demeester, A Surrogate Modeling and Adaptive Sampling Toolbox for Computer Based Design, Journal of Machine Learning Research 11 (2010) 2051 - 2055.

[29] H. Rabitz, O. F. Aliş, General foundations of high-dimensional model representations, Journal of Mathematical Chemistry 25 (2-3) (1999) 197-233, ISSN 0259-9791, doi: $\backslash$ bibinfo $\{$ doi $\}$ $\{10.1023 / A: 1019188517934\}$.

[30] G. Li, S. W. Wang, H. Rabitz, S. Wang, P. Jaffe, Global uncertainty assessments by high dimensional model representations (HDMR), Chemical Engineering Science 57 (21) (2002) 4445-4460, doi: bibinfo $\{$ doi $\}\{10.1016 / \mathrm{S} 0009-2509(02) 00417-7\}$, URL http://dx.doi.org/10.1016/S0009-2509(02)00417-7.

[31] G. Li, H. Rabitz, J. Hu, Z. Chen, Y. Ju, Regularized random-sampling high dimensional model representation (RSHDMR), Journal of Mathematical Chemistry 43 (3) (2008) 1207-1232, ISSN 0259-9791, doi: \bibinfo $\{$ doi $\}\{10.1007 /$ s10910-007-9250-x\}, URL http://dx.doi.org/10.1007/ s10910-007-9250-x.

[32] M. Krzywinski, N. Altman, Points of Significance: Visualizing samples with box plots, Nature Methods 11 (2014) $119-120$, doi: $\backslash$ bibinfo $\{$ doi $\}$ \{doi:10.1038/nmeth.2813\}.

[33] Minitab Express Support, Interpret the key results for boxplot, Webpage, URL http:// support.minitab.com/en-us/minitab-express/1/ help-and-how-to/basic-statistics/graphs/boxplot/ interpret-the-results/key-results/, 2015. 